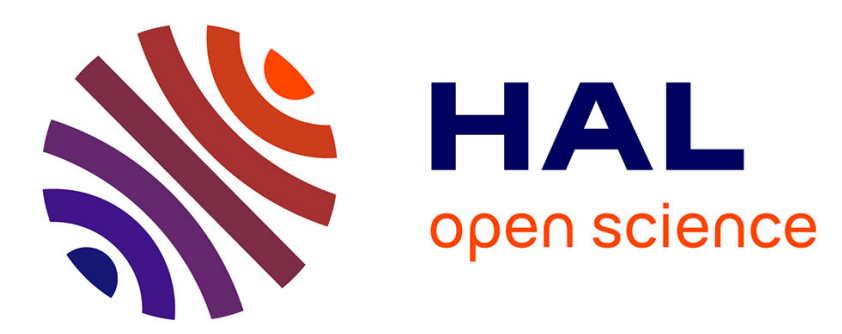

\title{
Méthode des intégrales de frontières pour les problèmes magnétiques à symétrie axiale et couplage avec la méthode des éléments finis
}

\author{
Xavier Brunotte, Jean-François Imhoff, Gérard Meunier
}

\section{To cite this version:}

Xavier Brunotte, Jean-François Imhoff, Gérard Meunier. Méthode des intégrales de frontières pour les problèmes magnétiques à symétrie axiale et couplage avec la méthode des éléments finis. Revue de Physique Appliquée, 1990, 25 (7), pp.613-626. 10.1051/rphysap:01990002507061300 . jpa-00246227

\section{HAL Id: jpa-00246227 https://hal.science/jpa-00246227}

Submitted on 1 Jan 1990

HAL is a multi-disciplinary open access archive for the deposit and dissemination of scientific research documents, whether they are published or not. The documents may come from teaching and research institutions in France or abroad, or from public or private research centers.
L'archive ouverte pluridisciplinaire HAL, est destinée au dépôt et à la diffusion de documents scientifiques de niveau recherche, publiés ou non, émanant des établissements d'enseignement et de recherche français ou étrangers, des laboratoires publics ou privés. 
Classification

Physics Abstracts

$41.10-41.10 \mathrm{D}-41.10 \mathrm{~F}-02.60-02.70$

\title{
Méthode des intégrales de frontières pour les problèmes magnétiques à symétrie axiale et couplage avec la méthode des éléments finis
}

\author{
Xavier Brunotte $\left({ }^{1}\right)$, Jean-François Imhoff $\left({ }^{2}\right)$ et Gérard Meunier $\left({ }^{2}\right)$ \\ (1) Laboratoire de Magnétisme du Navire, ENSIEG, BP 46, 38402 Saint Martin d'Hères Cedex, France \\ ${ }^{2}$ ) Laboratoire d'Electrotechnique de Grenoble, ENSIEG, BP 46, 38402 Saint Martin d'Hères Cedex, France
}

(Reçu le 3 juillet 1989, révisé le 14 mars 1990, accepté le 3 avril 1990)

\begin{abstract}
Résumé. - Le but de cet article est de faire part de notre expérience dans les couplages des Méthodes éléments finis-équations intégrales de frontières pour problèmes à symétrie axiale. Il rappelle d'abord la formulation intégrales de frontières pour le cas de géométries axiales en potentiel scalaire (total ou réduit). Il développe les formulations pour le potentiel vecteur avec des formulations en $\left(A_{\theta}\right)$ et en $\left(r A_{\theta}\right)$; cette dernière étant particulièrement intéressante dans l'optique du couplage. Il présente ensuite des exemples de couplages avec la méthode des éléments finis et les compare avec ceux donnés analytiquement ou par une méthode purement éléments finis.
\end{abstract}

Abstract. - This article presents a hybrid Finite Element/Boundary Element Analysis method for axisymmetric geometry. First, a boundary formulation for scalar potentials (total or reduced) is presented, then equations for vectorial potential are developed. The $\left(r A_{\theta}\right)$ formulation happens to be much more convenient for the coupling than the $\left(A_{\theta}\right)$ formulation. Some numerical results given by this hybrid method are presented and compared with results obtained using analytical and other numerical methods.

\section{Introduction.}

L'utilisation conjointe des éléments finis et des intégrales de frontières permet de regrouper leurs potentialités :

- les régions à problèmes physiques complexes (matériaux non linéaires par exemple) seront alors traitées par éléments finis;

- tandis que les problèmes à frontières ouvertes (prise en compte de l'infini) seront facilement traités par intégrales de frontières (perméabilité constante). On traitera aussi par intégrales de frontières l'interace en re eux pieces mo $i$ es en re e

par exemple), car de cette manière on n'a pas à faire évoluer le maillage au cours du temps.

Cette association "éléments finis-intégrales de frontières " a prouvé son intérêt à travers de nombreux articles [1-5], qui traitent de tels couplages, en particulier pour les problèmes bidimensionnels plans et tridimensionnels. Par contre, le couplage pour des problèmes axisymétriques n'est que rarement abordé en partie à cause de la complexité plus grande des intégrales de frontières.

Cet article développe les formulations intégrales en axisymétrique pour potentiels scalaires (total et réduit), puis potentiel vecteur avec le développement original en $\left(r A_{\theta}\right)$ que nous avons développé parallèlement à $\mathrm{L}$. Pichon $[6,7]$; enfin il présente quelques exemples de mise en œuvre du couplage.

On pourra noter que les formulations intégrales de frontières choisies sont basées sur l'identité de Green. D'autres formulations existent, basées en particulier sur la théorie des distributions. Pour le cas axisymétrique et comme le montre $[6,7]$, on peut mener de manière similaire le calcul des noyaux; ce qui conduit aussi à la définition des onctions notees ans a suite axi, axi, ${ }^{-} \operatorname{axi}$ vec $r A \cdots$

\section{Rappel sur les intégrales de frontières}

Une variable $V$ de classe $\mathrm{C}_{2}$, dont le Laplacien est nul dans une région peut s'exprimer en tout point $\mathbf{P}$ de cette région en fonction de $V$ et de sa dérivée normale sur la frontière suivant :

$$
c(\mathrm{P}) V(\mathrm{P})=-\int_{\mathrm{S}}\left(V(\mathrm{Q}) \frac{\mathrm{d} G}{\mathrm{~d} n}-G \frac{\mathrm{d} V}{\mathrm{~d} n}\right) \mathrm{d} S
$$


où $G$ est la fonction de Green :

$$
\begin{aligned}
G & =\frac{1}{4 \pi \mathrm{PQ}} \quad \text { en } 3 \mathrm{D} \\
G & =\frac{-\log (\mathrm{PQ})}{2 \pi} \quad \text { en } 2 \mathrm{D} .
\end{aligned}
$$

Dans le cas général, on ne connaît pas entièrement $V$ et sa dérivée normale sur les frontières. Pour les déterminer, on génère un maillage des frontières. $\mathrm{Ce}$ maillage permet comme dans la méthode des élé- ments finis de définir un ensemble $\left(W_{i}\right)$ de fonctions d'approximations pour la frontière. On peut alors chercher une solution approchée $V^{*}$ du potentiel $V$ et $\Psi^{*}$ de sa dérivée normale sur les frontières en écrivant :

$$
\begin{aligned}
& V^{*}=\sum_{i}^{N} V_{i} \cdot W_{i} \\
& \psi^{*}=\left(\frac{\mathrm{d} V}{\mathrm{~d} n}\right)^{*}=\sum_{i}^{N} \psi_{i} \cdot W_{i} .
\end{aligned}
$$

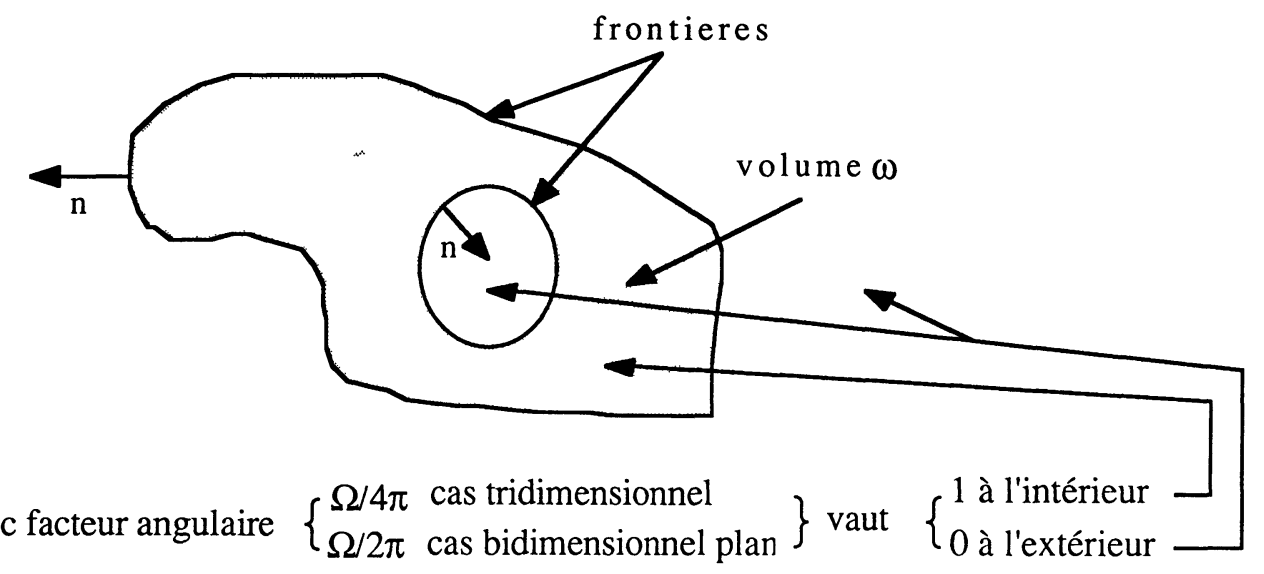

Fig. 1. - Illustration de l'équation intégrale de frontières (1)

[Illustration of boundary equation (1).]

Les $N$ valeurs $V_{i}$ (respectivement les $N \Psi_{i}$ ) sont par conséquent les valeurs de $V^{*}$ (resp. $\Psi^{*}$ ) aux nœuds correspondants. Pour trouver les valeurs inconnues, une solution est d'écrire (comme nous) $N$ équations intégrales (1) aux $N$ nœuds de la frontière,

$$
\begin{aligned}
c V_{i}=\sum_{j}^{N} V_{j} \int_{\text {front }} W_{j} \frac{\mathrm{d} G}{\mathrm{~d} n} \mathrm{~d} s- & \\
& -\sum_{j}^{N} \Psi_{j} \int_{\text {front }} W_{j} G \mathrm{~d} s .
\end{aligned}
$$

Le système linéaire ainsi obtenu est complété par soit les conditions aux limites, soit $N$ autres équations écrites sur cette même frontière pour les autres régions. On obtient ainsi un système linéaire (7) qu'il suffit alors de résoudre,

$$
\left[R_{i j}\right]\left[V_{j}\right]+\left[T_{i j}\right]\left[\psi_{j}\right]=\left[c V_{i}\right]
$$

où $\left[\mathrm{cV}_{i}\right]$ est une matrice diagonale.

Dans ce cas, comme le montre [8], il est intéressant de calculer numériquement le coefficient $\mathrm{c}$ par les équations de définition de l'angle solide (8) et (9):

- problème intérieur

$$
c=\int_{\mathrm{s}} \frac{\mathrm{d} G}{\mathrm{~d} n} \mathrm{~d} s
$$

— problème extérieur

$$
c=-1+\int_{\mathrm{s}} \frac{\mathrm{d} G}{\mathrm{~d} n} \mathrm{~d} s .
$$

\section{Remarques :}

- Au lieu d'écrire les $N$ équations aux nœuds de la frontière, il est envisageable de les écrire en $N$ points d'une surface extérieure au domaine; dans ce cas, le coefficient angulaire $c$ sous lequel le point $\mathbf{P}$ voit la région est nul, et on obtient le système matriciel :

$$
\left[R_{i j}\right]\left[V_{j}\right]+\left[T_{i j}\right]\left[\psi_{j}\right]=0 .
$$

L'inconvénient majeur de cette méthode est la nécessité de générer cette surface.

- Une troisième possibilité est de projeter l'équation (1) sur les fonctions $W_{i}$. Dans ce cas, $c$ étant une fonction de la frontière presque partout égale à $1 / 2$ sauf peut-être aux nœuds, on obtient le système :

$$
\begin{aligned}
\frac{1}{2} V_{i}\left\langle W_{i} \mid W_{k}\right\rangle & =\sum_{j}^{N} V_{j}\left\langle\int_{\text {front }} W_{j} \frac{\mathrm{d} G}{\mathrm{~d} n} \mathrm{~d} s \mid W_{k}\right\rangle \\
& -\sum_{j}^{N} \Psi_{j}\left\langle\int_{\text {front }} \frac{\mathrm{d} W_{j}}{\mathrm{~d} n} G \mathrm{~d} s \mid W_{k}\right\rangle
\end{aligned}
$$


où $\langle\mid\rangle$ est le produit scalaire de l'espace des fonctions intégrables de la frontière.

\section{Cas du potentiel scalaire en géométrie axiale.}

3.1 MODÈleS MAGNÉTOSTATIQUES SCALAIRES. En magnétostatique, sous condition d'existence d'une loi biunivoque (de type $B=\mu H$ ) constitutive du matériau, le potentiel $V$ duquel dérive le champ d'excitation $H$ en l'absence de courants, vérifie l'équation

$$
\operatorname{div}(\mu \operatorname{grad} V)=0
$$

qui dans l'air ou pour une région linéaire et isotrope devient

$$
\Delta V=0
$$

$V$ vérifie par conséquent l'équation (1), que l'on préfère écrire en faisant intervenir la composante normale du champ magnétique d'induction :

$c(\mathrm{P}) V(\mathrm{P})=-\int_{\mathrm{s}}\left(V(\mathrm{Q}) \frac{\mathrm{d} G}{\mathrm{~d} n}-G \frac{B_{\mathrm{n}}}{\mu}\right) \mathrm{d} S$.
Cette dernière expression présente en effet l'avantage d'assurer facilement la conservation de la composante normale de $B$.

En présence de courants, ou d'un champ source extérieur d'origine quelconque, le potentiel $V_{\text {red }}$ créé par la matière vérifie aussi l'équation (13), et par conséquent l'équation (15)

$$
\begin{aligned}
c(\mathrm{P}) V_{\mathrm{red}}(\mathrm{P}) & = \\
- & \int_{\mathrm{s}}\left(V_{\mathrm{red}}(\mathrm{Q}) \frac{\mathrm{d} G}{\mathrm{~d} n}-G \frac{B_{\mathrm{n}_{\mathrm{red}}}}{\mu}\right) \mathrm{d} S
\end{aligned}
$$

que l'on préfère écrire (toujours dans le but d'assurer facilement la conservation de la composante normale du champ total magnétique d'induction)

$$
\begin{aligned}
& c \cdot V_{\text {red }}(\mathrm{P})= \\
& -\int_{\mathrm{s}}\left(V_{\mathrm{red}}(\mathrm{Q}) \frac{\mathrm{d} G}{\mathrm{~d} n}-G\left(\frac{B_{\mathrm{n}_{\mathrm{tot}}}}{\mu}-H_{\mathrm{n}_{0}}\right)\right) \mathrm{d} S
\end{aligned}
$$

$B_{\mathrm{n}_{\text {tot }}}$ étant la nouvelle inconnue sur la frontière, et $H_{\mathrm{n}_{0}}$ le champ source.

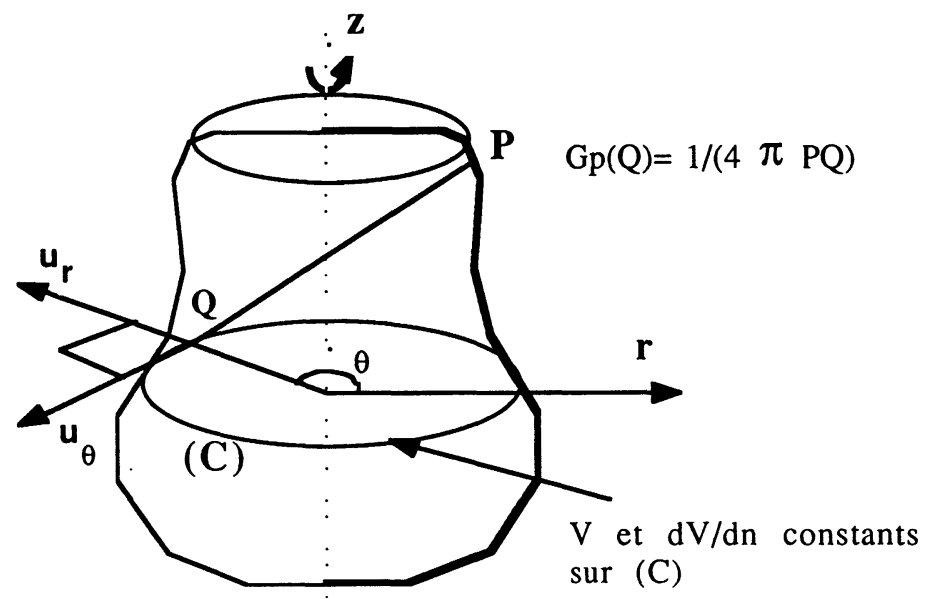

Fig. 2. - Problème axisymétrique typique.

[Typical axisymmetric problem.]

3.2 Formulation AXISYMÉTRIQUE. - Un proeme $p$ ysique a symetrie axia e es un pro eme tridimensionnel pour lequel les grandeurs physiques intéressantes (ici $V$ et $B_{\mathrm{n}}$ ou $\mathrm{d} V / \mathrm{d} n$ ) sont indépendantes de l'angle $\boldsymbol{\theta}$.

La fonction de Green du point $P$ calculée au point $Q$ (ainsi que sa dérivée normale en $Q$ ) dépendant par contre de l'angle $\theta$, il est intéressant de préintégrer les noyaux $G$ et $\mathrm{d} G / \mathrm{d} n$ sur (C). L'équation intégrale (14) s'écrit alors (cf. [9]) :

$c(\mathrm{P}) V(\mathrm{P})=-\int_{\mathrm{L}}\left(V(\mathrm{Q}) G_{\mathrm{axi}}^{\prime}-G_{\mathrm{axi}} \frac{B_{\mathrm{n}}}{\mu}\right) \mathrm{d} \ell$ où $G_{\text {axi }}$ et $G_{\text {axi }}^{\prime}$ s'expriment par

$$
G_{\mathrm{axi}}=\int_{0}^{2 \pi} G r \mathrm{~d} \theta
$$

et

$$
G_{\mathrm{axi}}^{\prime}=\int_{0}^{2 \pi} \frac{\mathrm{d} G}{\mathrm{~d} n} r \mathrm{~d} \theta
$$

Le calcul des intégrales (18) et (19) donne avec les notations de la figure 3

$$
G_{\mathrm{axi}}=\frac{r}{\pi D} J_{1}\left(k^{2}\right)
$$




$$
\begin{aligned}
G_{\mathrm{axi}}^{\prime}= & -\frac{n_{r}}{2 \pi D} J_{1}\left(k^{2}\right)+ \\
& +\frac{\left(h^{2}+R^{2}-r^{2}\right) \cdot n_{r}+2 h \cdot r \cdot n_{z}}{2 \pi D D^{\prime 2}} J_{2}\left(k^{2}\right)
\end{aligned}
$$

où $J_{1}$ et $J_{2}$ sont les intégrales elliptiques complètes de première et deuxième espèce

$$
\begin{aligned}
& J_{1}\left(k^{2}\right)=\int_{0}^{\pi / 2}\left(1-k^{2} \sin ^{2} \theta\right)^{-\frac{1}{2}} \mathrm{~d} \theta \\
& J_{2}\left(k^{2}\right)=\int_{0}^{\pi / 2}\left(1-k^{2} \sin ^{2} \theta\right)^{\frac{1}{2}} \mathrm{~d} \theta
\end{aligned}
$$

avec

$$
k^{2}=4 \frac{r \cdot R}{D^{2}}
$$

Remarque. - Le coefficient $c$ est calculé numériquement par

$$
\begin{gathered}
c=\int_{\mathrm{L}} G_{\mathrm{axi}}^{\prime} \mathrm{d} \ell \text { pour un problème intérieur } \\
c=-1+\int_{\mathrm{L}} G_{\mathrm{axi}}^{\prime} \mathrm{d} \ell \\
\text { pour un problème extérieur }
\end{gathered}
$$

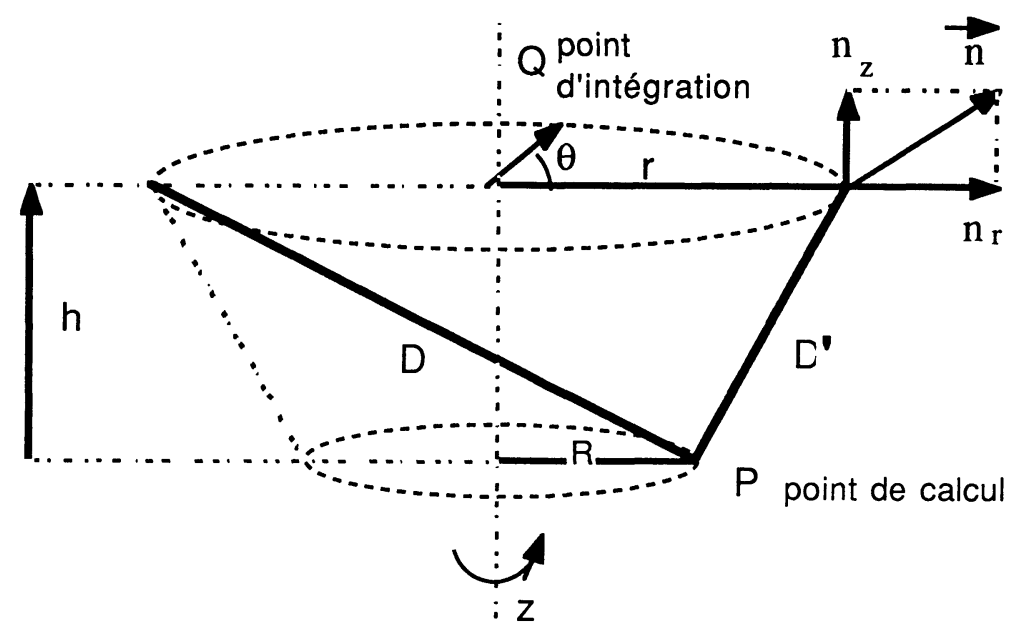

Fig. 3. - Notations.

[Notations.]

\section{Cas du potentiel vecteur en géométrie axiale.}

Le potentiel vecteur, duquel dérive le champ magnétique $B$ vérifie l'équation sous condition d'existence d'une loi d'aimantation $H=\nu B$ (où $\nu$ est la reluctivité et $J$ la densité de courant)

$$
\operatorname{rot}(\nu \operatorname{rot}(\mathbf{A}))=\mathbf{J} \text {. }
$$

En l'absence de courants et pour des régions à reluctivité constante et isotrope, (24) se transforme en l'équation vectorielle (25)

$$
\Delta \mathbf{A}=\mathbf{0} .
$$

La formulation intégrale de frontières en potentiel vecteur ne pose pas de problèmes particuliers en $2 \mathrm{D}$ cartésien, car alors l'équation vectorielle $\Delta \mathbf{A}=\mathbf{0}$ implique la nullité du Laplacien de l'unique composante de $A\left(\Delta A_{z}=0\right)$. On peut par conséquent écrire l'équation (1) pour $A_{z}$.

Par contre, une formulation intégrale de frontières est plus difficile à obtenir pour un problème axisymétrique, car l'équation $\Delta \mathbf{A}=\mathbf{0}$ n'implique pas
$\Delta A_{\theta}=0$ (le vecteur $A$ s'exprime par $\mathbf{A}=A_{\theta} \cdot \mathbf{u}_{\theta}$ ). Une méthode simple est de remarquer que les Laplaciens des composantes $A_{x}$ et $A_{y}$ de $A$ sont nuls dans un repère cartésien tridimensionnel fixe. On peut donc écrire l'équation (1) pour $A_{x}$ ou $A_{y}$. On a en particulier :

$$
c \cdot A_{y}=-\int_{\mathrm{s}}\left(A_{y} \frac{\mathrm{d} G}{\mathrm{~d} n}-G \frac{\mathrm{d} A_{y}}{\mathrm{~d} n}\right) \mathrm{d} S .
$$

En coordonnées cylindriques $(r, \theta, z), A_{y}$ s'exprime par

$$
A_{y}=A_{r} \sin \theta+A_{\theta} \cos \theta .
$$

Or pour un problème à symétrie axiale, $A_{r}$ est égale à zéro et $A_{\theta}$ est indépendant de $\theta$ (on a $\left.A_{\theta}(r, \theta, z)=A_{\theta}(r, 0, z)\right)$. Par conséquent, en écrivant l'équation (26) en un point du plan $\theta=0$, on obtient

$$
c \cdot A_{\theta}=-\int_{\mathrm{s}}\left(A_{\theta} \cos \theta \frac{\mathrm{d} G}{\mathrm{~d} n}-G \frac{\mathrm{d}\left(A_{\theta} \cos \theta\right)}{\mathrm{d} n}\right) \mathrm{d} S .
$$


De plus

$$
\frac{\mathrm{d}\left(A_{\theta} \cos \theta\right)}{\mathrm{d} n}=\frac{\mathrm{d} A_{\theta}}{\mathrm{d} n} \cdot \cos \theta .
$$

On peut alors écrire de la même manière qu'en 3

$$
c \cdot A_{\theta}=-\int_{\mathrm{L}}\left(A_{\theta} G_{\text {axi vec }}^{\prime}-\frac{\mathrm{d} A_{\theta}}{\mathrm{d} n} G_{\text {axi vec }}\right) \mathrm{d} \boldsymbol{\ell}
$$

où

$$
G_{\text {axi vec }}=\int_{0}^{2 \pi} G \cos \theta r \mathrm{~d} \theta
$$

et

$$
G_{\mathrm{axi} \mathrm{vec}}^{\prime}=\int_{0}^{2 \pi} \frac{\mathrm{d} G}{\mathrm{~d} n} \cos \theta r \mathrm{~d} \theta
$$

Les calculs montrent :

$$
G_{\mathrm{axi} \mathrm{vec}}=\frac{D}{4 \pi R}\left[\left(2-k^{2}\right) J_{1}\left(k^{2}\right)-2 J_{2}\left(k^{2}\right)\right]
$$

et

$$
\begin{aligned}
G_{\mathrm{axi} \mathrm{vec}}^{\prime}= & \frac{h n_{z}}{2 \pi D R}\left[-J_{1}\left(k^{2}\right)+\frac{R^{2}+r^{2}+h^{2}}{D^{\prime 2}} J_{2}\left(k^{2}\right)\right] \\
& +\frac{r n_{r}}{2 \pi D R}\left[-\frac{R^{2}+h^{2}}{r^{2}} J_{1}\left(k^{2}\right)\right. \\
& \left.+\left(\frac{R^{2}-r^{2}-h^{2}}{D^{\prime 2}}+\frac{D^{2}}{r^{2}}\right) J_{2}\left(k^{2}\right)\right]
\end{aligned}
$$

\section{Remarques.}

- En écrivant l'équation (1) pour $A_{x}$ à la place de $A_{y}$, on aurait obtenu l'équation

$0=-\int_{\mathrm{s}}\left(A_{\theta} \sin \theta \frac{\mathrm{d} G}{\mathrm{~d} n}-\frac{\mathrm{d}\left(A_{\theta}\right)}{\mathrm{d} n} G \sin \theta\right) \mathrm{d} S$

dont l'inconvénient est de ne pas donner le champ à l'intérieur du domaine.

- Nous avons retrouvé l'équation (30) complétée par (31) et (32), en partant, comme certains auteurs de l'identité vectorielle (36), analogue à la seconde identité de Green dont découle (1)

$$
\begin{aligned}
& {[\mathbf{A} \cdot \operatorname{rot}(\operatorname{rot} \mathbf{B})-\mathbf{B} \cdot \operatorname{rot}(\operatorname{rot} \mathbf{A})] \mathrm{d} v=} \\
& \quad=\int_{\mathrm{S}}[\mathbf{A} \wedge \operatorname{rot} \mathbf{B}-\mathbf{B} \wedge \operatorname{rot} \mathbf{A}] \cdot \mathrm{d} \mathbf{S} .
\end{aligned}
$$

Les calculs que nécessite cette méthode sont cependant plus complexes (elle nécessite en particulier la définition d'une fonction vectorielle de Green) et ne présentent pas d'intérêt à être développés ici.

- Le terme $c$ dans l'expression (30) est toujours calculé par les relations (22) ou (23).

\section{Formulation en $\left(\boldsymbol{r} \boldsymbol{A}_{\theta}\right)$.}

Dans le code de calcul de Fluxlab, la formulation éléments finis axisymétrique choisie fait intervenir de manière classique la variable $\left(r A_{\theta}\right)$ (cf. paragraphe 7). Nous avons donc transformé l'équation (30) en écrivant (avec les notations de la Fig. 3)

$$
\begin{gathered}
A_{\theta}=\frac{\left(r A_{\theta}\right)}{r} \\
c A_{\theta}=c \frac{\left(R A_{\theta}\right)}{R} \\
\frac{\mathrm{d} A_{\theta}}{\mathrm{d} n}=\frac{1}{\mathrm{r}} \frac{\mathrm{d}\left(r A_{\theta}\right)}{\mathrm{d} n}=\frac{1}{r^{2}} \frac{\mathrm{d} r}{\mathrm{~d} n}\left(r A_{\theta}\right)= \\
=\frac{1}{\mathrm{r}} \frac{\mathrm{d}\left(r A_{\theta}\right)}{\mathrm{d} n}-\frac{n_{r}}{r^{2}}\left(r A_{\theta}\right) .
\end{gathered}
$$

On obtient alors

$$
\begin{aligned}
c \cdot\left(R A_{\theta}\right)=-\int_{\mathrm{L}}\left[\left(r A_{\theta}\right) G_{\mathrm{axi} \text { vec } r A}^{\prime}-\right. \\
\\
\left.\quad-\frac{\mathrm{d}\left(r A_{\theta}\right)}{\mathrm{d} n} G_{\text {axi vec } r A}\right] \mathrm{d} \ell
\end{aligned}
$$

avec

$$
\begin{aligned}
& G_{\text {axi vec } R A}=\frac{R}{r} G_{\text {axi vec }} \\
& G_{\text {axi vec } R A}^{\prime}=\frac{R}{r} G_{\text {axi vec }}^{\prime}-\frac{n_{r} R}{r^{2}} G_{\text {axi vec }} .
\end{aligned}
$$

De (33), (34), (41) et (42) on déduit :

$$
G_{\text {axi vec } R A}=\frac{D}{4 \pi r}\left[\left(2-k^{2}\right) \cdot J_{1}-2 . J_{2}\right]
$$

et

$$
\begin{array}{r}
G_{\mathrm{axi} \text { vec } R A}^{\prime}=\frac{h n_{z}}{2 \pi D r}\left[-J_{1}+\frac{R^{2}+r^{2}+h^{2}}{D^{\prime 2}} J_{2}\right]+ \\
+\frac{n_{r}}{2 \pi D}\left[J_{1}+\frac{R^{2}-r^{2}-h^{2}}{D^{\prime 2}} J_{2}\right] .
\end{array}
$$

\section{Remarques.}

- On peut remarquer que (44) est relativement plus simple que (34). Mais l'intérêt essentiel de la formulation en $\left(r A_{\theta}\right)$ s'explique par la simplification dus au couplage (cf. paragraphe 7).

- Dans la pratique, pour faciliter le couplage et assurer la conservation de la composante tangentielle du champ magnétique d'excitation $H$, nous avons introduit la variable $\left(r H_{\mathrm{t}}\right)$ dans $(40)$, comme nous avions introduit $B_{\mathrm{n}}$ en 3 ; ce qui donne :

$$
\begin{aligned}
c \cdot\left(R A_{\theta}\right)=-\int_{\mathrm{L}}\left[\left(r A_{\theta}\right) G_{\text {axi vec } r A}^{\prime}-\right. & \\
& \left.-\frac{\left(r H_{\mathrm{t}}\right)}{\nu} G_{\text {axi vec } r A}\right] \mathrm{d} \boldsymbol{\ell} .
\end{aligned}
$$




\section{Couplage en potentiels scalaires et exemple.}

6.1 FORMULATION ÉlÉMENTS FINIS POTENTIEL TOTAL. - La méthode des éléments finis est basée sur un maillage en éléments finis du domaine. Ce maillage permet, dans le cadre de la méthode de Galerkin, la construction d'un ensemble de fonctions poids $\left(W_{i}\right)$. L'équation $\operatorname{div} \mu \operatorname{grad} V=0$ (12), que l'on cherche à résoudre implique l'ensemble d'équations (46) pour tout $W_{i}$

$$
\int_{\text {domaine }} W_{i} \operatorname{div}(\mu \operatorname{grad} V) \mathrm{d} v=0
$$

ensemble d'équations, qui intégrées par partie, donnent pour tout $W_{i}$

$$
\begin{aligned}
\int_{\text {domaine }} \operatorname{grad} W_{i}(\mu \operatorname{grad} V) \mathrm{d} v= & \\
= & -\int_{\text {contour }} W_{i} B_{\mathrm{n}} \mathrm{d} S .
\end{aligned}
$$

A partir des équations (47), on cherche une approximation (48) de $V$, qui conduit à la résolution d'un système matriciel (49)

$$
\begin{gathered}
V^{*}=\sum_{j} V_{j} W_{j} \\
{\left[S_{i j}\right]\left[V_{j}\right]=\left[R_{i}\right] .}
\end{gathered}
$$

Remarques.

- Le terme $\mu$ peut être dans un contexte éléments finis un tenseur (anisotropie de la matière) et, par une méthode de type Newton-Raphson, dépendant de $H$ (matériaux non linéaires, en particulier au coude de saturation).

- A l'interface entre deux régions de perméabilités différentes, les intégrales de contour calculées pour une fonction de projection $W_{i}$ sur les deux régions, sont égales (conservation de la composante normale de l'induction). L'équation (47) conduit alors à

$$
\begin{aligned}
\int_{\text {domaine } 1} & \operatorname{grad} W_{i}\left(\mu_{1} \operatorname{grad} V\right) \mathrm{d} v+ \\
+ & \int_{\text {domaine } 2} \operatorname{grad} W_{i}\left(\mu_{2} \operatorname{grad} V\right) \mathrm{d} v=0 .
\end{aligned}
$$

- Dans une méthode uniquement éléments finis, le terme de contour de la matrice $\left[R_{i}\right]$ est nul pour presque tous les $W_{i}$; quand il n'est pas nul, il est donné par les conditions aux limites.

6.2 FORMUlation ÉlÉMENTS FINIS POTENTIEL RÉDUIT. - En potentiel réduit et si la région est linéaire et isotrope, le terme source se ramène à une intégrale surfacique sur les interfaces entre différentes régions. L'équation équivalente à (50) s'écrit alors

$$
\begin{aligned}
& \int_{\text {dom 1 }} \operatorname{grad} W_{i}\left(\mu_{1} \operatorname{grad} V_{\mathrm{red}}\right) \mathrm{d} v+ \\
& +\int_{\operatorname{dom} 2} \operatorname{grad} W_{i}\left(\mu_{2} \operatorname{grad} V_{\mathrm{red}}\right) \mathrm{d} v= \\
& =\int_{\text {inter }} W_{i}\left(\mu_{2}-\mu_{1}\right) H_{\text {on }} \mathrm{d} S
\end{aligned}
$$

Si la région est non linéaire, il faut intégrer en volume le terme source suivant

$$
\begin{aligned}
\int_{\text {dom }} \operatorname{grad} W_{i}\left(\mu \operatorname{grad} V_{\text {red }}\right) \mathrm{d} v= \\
=-\int_{\operatorname{dom}} \operatorname{grad} W_{i}\left(\mu \mathbf{H}_{0}\right) \mathrm{d} v \\
-\int_{\text {contour }} W_{i} B_{\mathrm{n} \text { tot }} \mathrm{d} S .
\end{aligned}
$$

6.3 CAS AXISYMÉTRIQUE. - La formulation axisymétrique ne pose aucun problème particulier pour les potentiels scalaires. Les termes des matrices $\left[S_{i j}\right]$ et $\left[R_{i}\right]$ valent alors

$$
\begin{aligned}
S_{i j} & =\int_{\mathrm{s}} \mu\left(\frac{\mathrm{d} W_{i}}{\mathrm{~d} r} \frac{\mathrm{d} W_{j}}{\mathrm{~d} r}+\frac{\mathrm{d} W_{i}}{\mathrm{~d} z} \frac{\mathrm{d} W_{j}}{\mathrm{~d} z}\right) 2 \pi r \mathrm{~d} \tau \\
R_{i} & =-\int_{\mathrm{L}} B_{\mathrm{n}} W_{i} 2 \pi r \mathrm{~d} \ell
\end{aligned}
$$

où $r$ est le rayon au point courant d'intégration.

6.4 Couplage. - L'équation (47), de laquelle on tire le système matriciel (49), fait intervenir le potentiel sur toute la région et sa dérivée normale sur sa frontière. Le contour de la région se sépare en une interface avec la région que l'on traite par intégrales de frontières, et le reste du contour. On obtient alors (55), où $B_{\mathrm{n}}$ est inconnue sur l'interface et $V$ dans le domaine

$$
\begin{aligned}
\int_{\text {domaine }} & \operatorname{grad} W_{i}(\mu \operatorname{grad} V) \mathrm{d} v+ \\
+ & +\int_{\text {inter }} W_{i} B_{\mathrm{n}} \mathrm{d} S=-\int_{\text {contour }} W_{i} B_{\mathrm{n}} \mathrm{d} S
\end{aligned}
$$

d'où l'on tire le système (56)

$$
\left[S_{i j}\right]\left[V_{j}\right]+\left[U_{i j}\right]\left[B_{\mathrm{n}_{j}}\right]=\left[Q_{i}\right]
$$

que l'on résout simultanément avec (7)

$$
\left[R_{i j}\right]\left[V_{j}\right]-\left[c V_{i}\right]+\left[T_{i j}\right]\left[B_{\mathrm{n}_{j}}\right]=0 .
$$

En potentiel réduit, on intègre le terme source du côté éléments finis [10].

Remarque. - Une autre technique de résolution du système couplé (7) et (56) est d'exprimer la matrice $\left[B_{\mathrm{n},}\right]$ en fonction de $\left[T_{i j}\right]^{-1},\left[R_{i j}\right],\left[V_{i}\right]$, à partir de 
(7) et de la remplacer dans (56); l'intérêt est que la matrice que l'on obtient est symétrisable.

6.5 EXEMPLE: SPHÈRE FERROMAGNÉTIQUE DANS UN CHAMP UNIFORME. COMPARAISON AVEC LA
SOLUTION ANALYTIQUE. - La sphère (de rayon $a=1 \mathrm{~m}$ ) est constituée d'un matériau linéaire de perméabilité 10 (Fig. 4). Elle est plongée dans un champ uniforme de $21 \times 10^{-5} \mathrm{~T}$ ou encore $16,71 \mathrm{~A} / \mathrm{m}$.

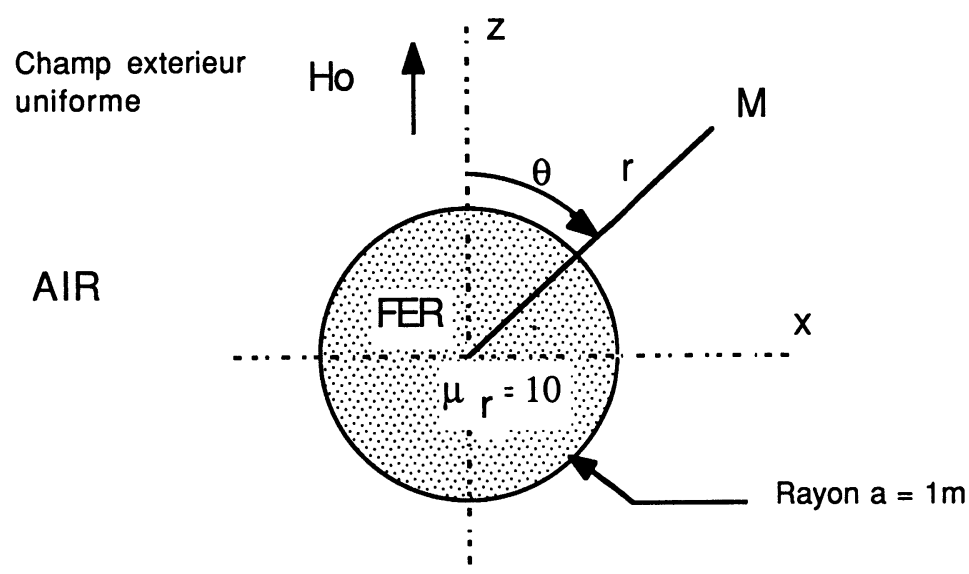

Fig. 4. - Cas de la sphère plongée dans un champ uniforme.

[Case of a sphere in an uniform magnetic field.]

La solution analytique donnée par [11] s'écrit au point $\mathrm{M}$,

$$
V_{\text {total }}=\left(A \frac{a^{3}}{r^{3}}-1\right) H_{0} r \cos \theta
$$

où

$$
A=\frac{\mu_{\mathrm{r}}-\mu_{0}}{\mu_{\mathrm{r}}+2 \mu_{0}}=\frac{3}{4}
$$

ce qui donne pour le potentiel réduit qui est la variable d'état choisie

$$
V_{\text {réduit }}=A \frac{a^{3}}{r^{3}} H_{0} r \cos \theta=\frac{3}{4} H_{0} \frac{z}{r^{3}} .
$$

Pour la résolution numérique, on n'entre qu'un quart de la géométrie, à cause de l'antisymétrie par rapport à l'axe des $x$, et de la symétrie radiale suivant $z$ (Fig. 5). La figure 6 montre les équipoten-

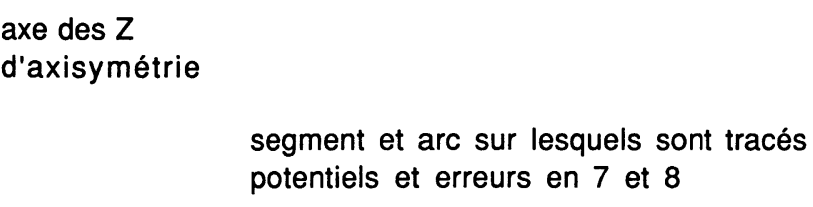

EIF
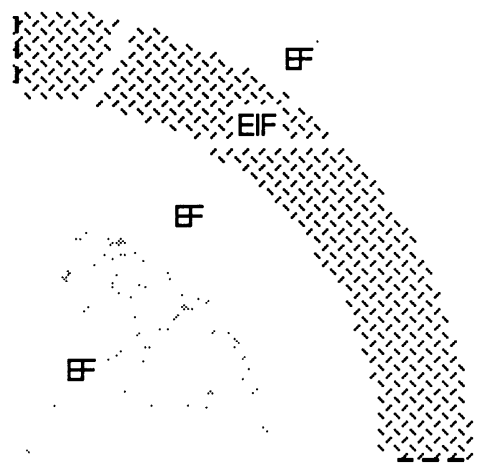

Air traité par E.F.

$1 \mathrm{~m}$

$1.4 \mathrm{~m} \quad 1.8 \mathrm{~m} \quad 2 \mathrm{~m}$

axe des $X$ d'antisymétrie

Fig. 5. - Problème modélisé.

[Modelled problem.] 


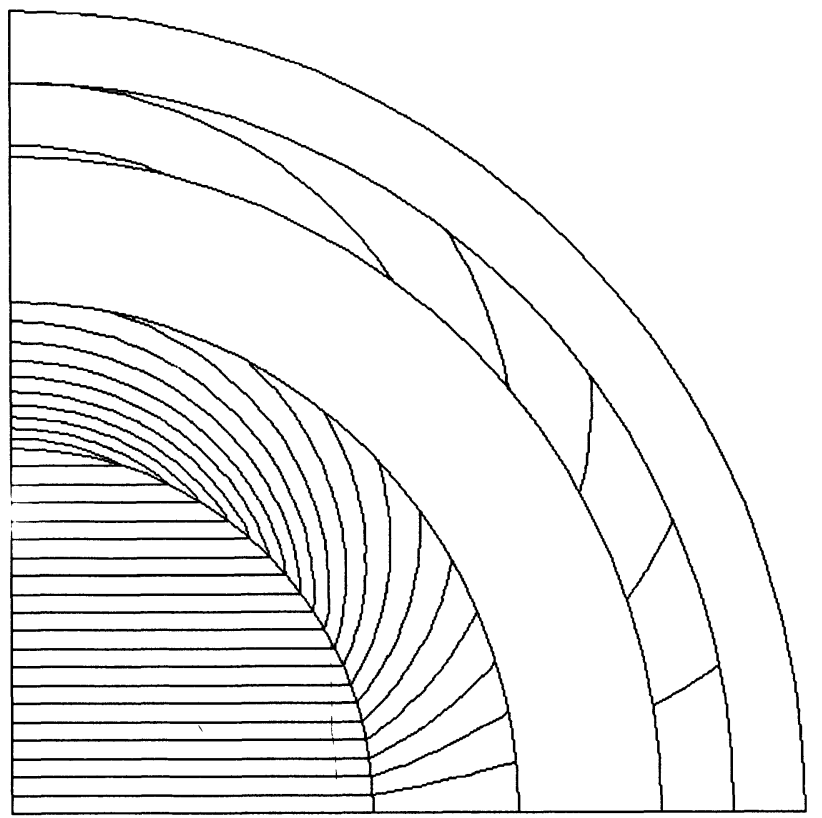

Fig. 6. - Equipotentielles dans les régions Eléments Finis.

[Equipotential lines in the Finite Element areas.] tielles dans les régions traitées par éléments finis. La figure 7 donne le potentiel sur le segment de droite $\left[\left(1,4,90^{\circ}\right) ;\left(1,4,5^{\circ}\right)\right]$ en coordonnées polaires, et l'erreur absolue multipliée par cent sur ce segment ; tandis que la figure 8 donne le potentiel sur l'arc de cercle de rayon 1,9 et donne l'erreur relative en pourcentage.

\section{Couplage en potentiel vecteur et exemple.}

7.1 FORMULATION ÉLÉMENTS FINIS. - On veut résoudre l'équation vectorielle $\operatorname{rot}(\nu \operatorname{rot}(\mathbf{A}))=\mathbf{J}$, qu'on projette sur des vecteurs fonctions poids

$$
\int_{\mathrm{vol}} \mathbf{W}_{i} \cdot \operatorname{rot}(\nu \operatorname{rot} \mathbf{A}) \mathrm{d} v=\int_{\mathrm{vol}} \mathbf{W}_{i} \cdot \mathbf{J} \mathrm{d} v .
$$

En l'intégrant par partie, on obtient comme en 6, des termes volumiques et surfaciques

$$
\begin{aligned}
& \int_{\mathrm{vol}}\left(\operatorname{rot} \mathbf{W}_{i}\right) \cdot(\nu \operatorname{rot} \mathbf{A}) \mathrm{d} v= \\
& \quad=\int_{\mathrm{vol}} \mathbf{W}_{i} \cdot \mathbf{J} \mathrm{d} v-\int_{\mathrm{surf}} \mathbf{W}_{i} \wedge(\nu \operatorname{rot} \mathbf{A}) \mathrm{d} \mathbf{S} .
\end{aligned}
$$

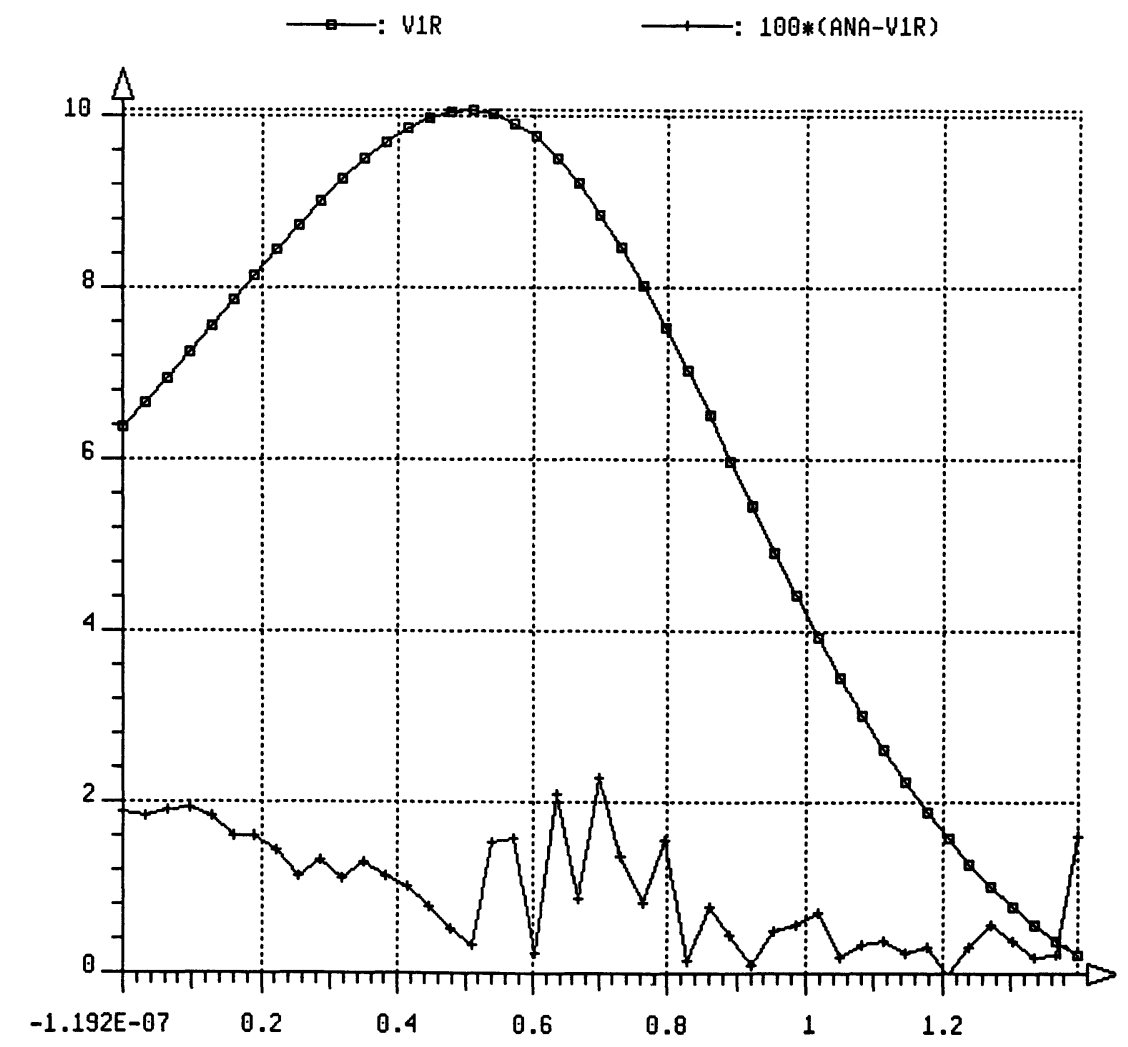

FLUXLAB

Fig. 7. - Potentiel et erreurs absolues $(\times 100)$ sur le segment.

[Potential and absolute errors $(\times 100)$ on the segment.] 


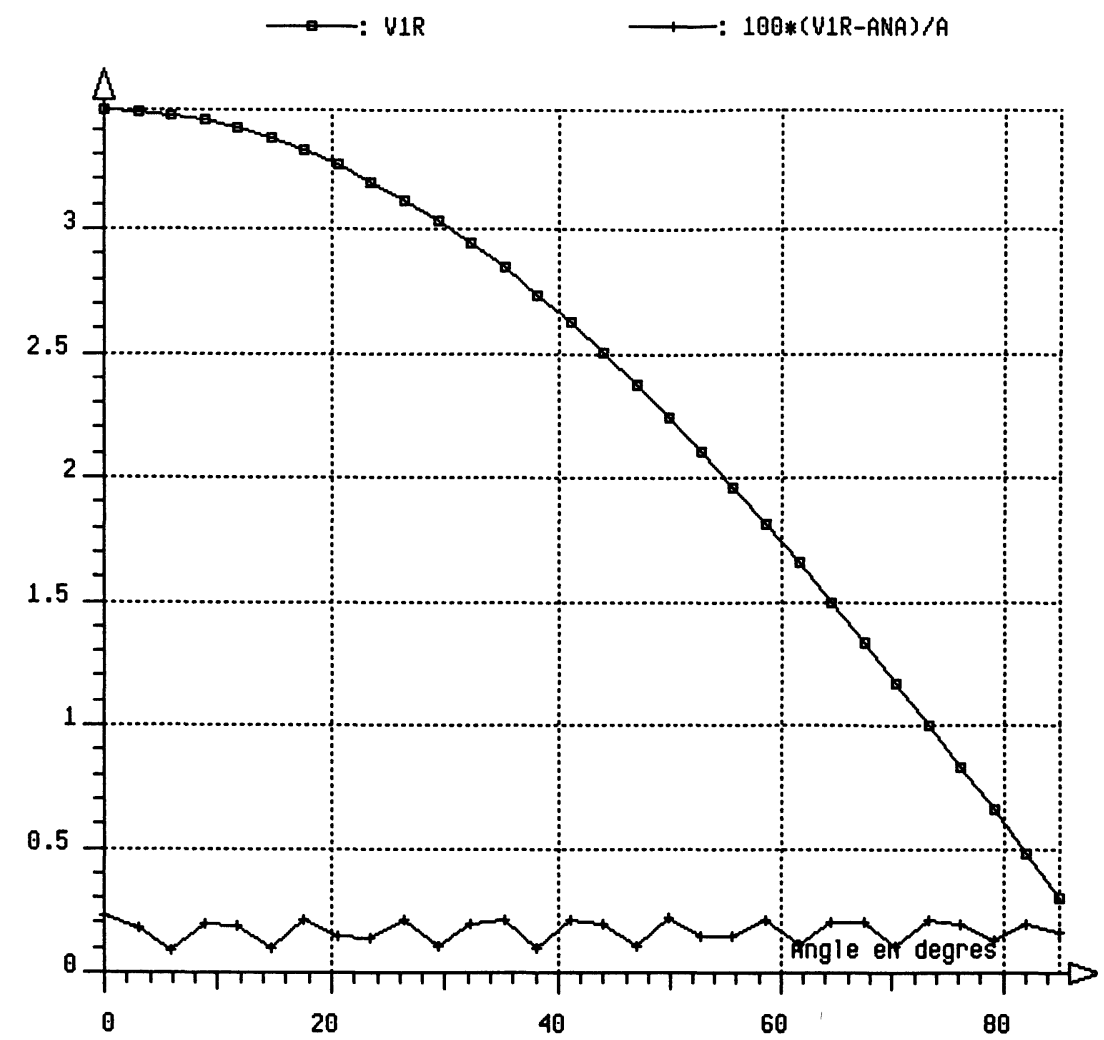

FLUXLAB

Fig. 8. - Potentiel et erreurs relatives $(\times 100)$ sur l'arc.

[Potential and relative errors $(\times 100)$ on the arc.]

En 2d cartésien (géométrie infiniment longue dans la direction $Z$ ), la seule composante non nulle du vecteur $A$ est $A_{Z}$. On choisit l'axe $Z$ comme direction des vecteurs fonctions de poids, ce qui donne un ensemble d'équations (60)

$$
\begin{aligned}
\int_{\mathrm{dom}} \nu\left(\frac{\mathrm{d} W_{i}}{\mathrm{~d} x}\right. & \left.\frac{A_{z}}{\mathrm{~d} x}+\frac{\mathrm{d} W_{i}}{\mathrm{~d} y} \frac{A_{z}}{\mathrm{~d} y}\right) \mathrm{d} \tau= \\
& =\int_{\mathrm{dom}} W_{i} J_{z} \mathrm{~d} \tau-\int_{\text {cont }} W_{i} H_{\mathrm{t}} \mathrm{d} c
\end{aligned}
$$

qu'on résume par

$$
\begin{aligned}
\int_{\mathrm{dom}} \nu\left(\operatorname{grad} W_{i} \operatorname{grad} A_{z}\right) \mathrm{d} \tau= \\
=\int_{\text {dom }} W_{i} J_{z} \mathrm{~d} \tau-\int_{\text {const }} W_{i} H_{\mathrm{t}} \mathrm{d} c .
\end{aligned}
$$

7.2 FORMUlATION ÉlÉMENTS FINIS. GÉOMÉTRIE AXIALE. - Pour un problème axisymétrique pour lequel $A$ est axial, on choisit comme vecteurs fonctions poids des vecteurs axiaux. En exprimant les rotationnels en coordonnées cylindriques dans (59), on obtient

$$
\begin{aligned}
& \int_{\mathrm{dom}} \nu\left(\frac{\mathrm{d} W_{i}}{\mathrm{~d} r} \frac{A_{\theta}}{\mathrm{d} r}+\frac{\mathrm{d} W_{i}}{\mathrm{~d} z} \frac{A_{\theta}}{\mathrm{d} z}+\frac{\mathrm{d} W_{i}}{\mathrm{~d} r} \frac{A_{\theta}}{r}+\frac{W_{i}}{r} \frac{\mathrm{d} A_{\theta}}{\mathrm{d} r}+\right. \\
& \left.+\frac{W_{i} A_{\theta}}{r^{2}}\right) 2 \pi r \mathrm{~d} \tau=\int_{\text {dom }} W_{i} J_{\theta} 2 \pi r \mathrm{~d} \tau \\
& -\int_{\text {cont }} W_{i}\left(\frac{\mathrm{d} A_{\theta}}{\mathrm{d} n}+n_{r} \frac{A_{\theta}}{r}\right) 2 \pi r \mathrm{~d} c .
\end{aligned}
$$

En introduisant la variable $r A$ ainsi que les fonctions poids $W_{i}^{*}=\left(r W_{i}\right)$, où $r$ est le rayon au point d'intégration, on obtient les équations

$$
\begin{aligned}
& \underset{\operatorname{dom}}{\nu} \frac{\mathrm{d} W_{i}^{*}}{\mathrm{~d} r} \frac{\mathrm{d} r A}{\mathrm{~d} r}+\frac{\mathrm{d} W_{i}^{*} \mathrm{~d} r A}{\mathrm{~d} z} \frac{?}{\mathrm{~d} z} \quad \tau= \\
& =\int_{\mathrm{dom}} W_{i}^{*} J_{\theta} 2 \pi \mathrm{d} \tau-\int_{\mathrm{cont}} W_{i}^{*}\left(r H_{\mathrm{t}}\right) \frac{2 \pi}{r} \mathrm{~d} c .
\end{aligned}
$$

7.3 Couplage. - Le couplage des deux méthodes ne présente, à partir des formulations $(r A)$ éléments finis et intégrales de frontières et par rapport au potentiel scalaire, aucune difficulté supplémentaire. La seule différence est qu'une équation intégrale de frontière calculée en un nœud de l'axe donne une 


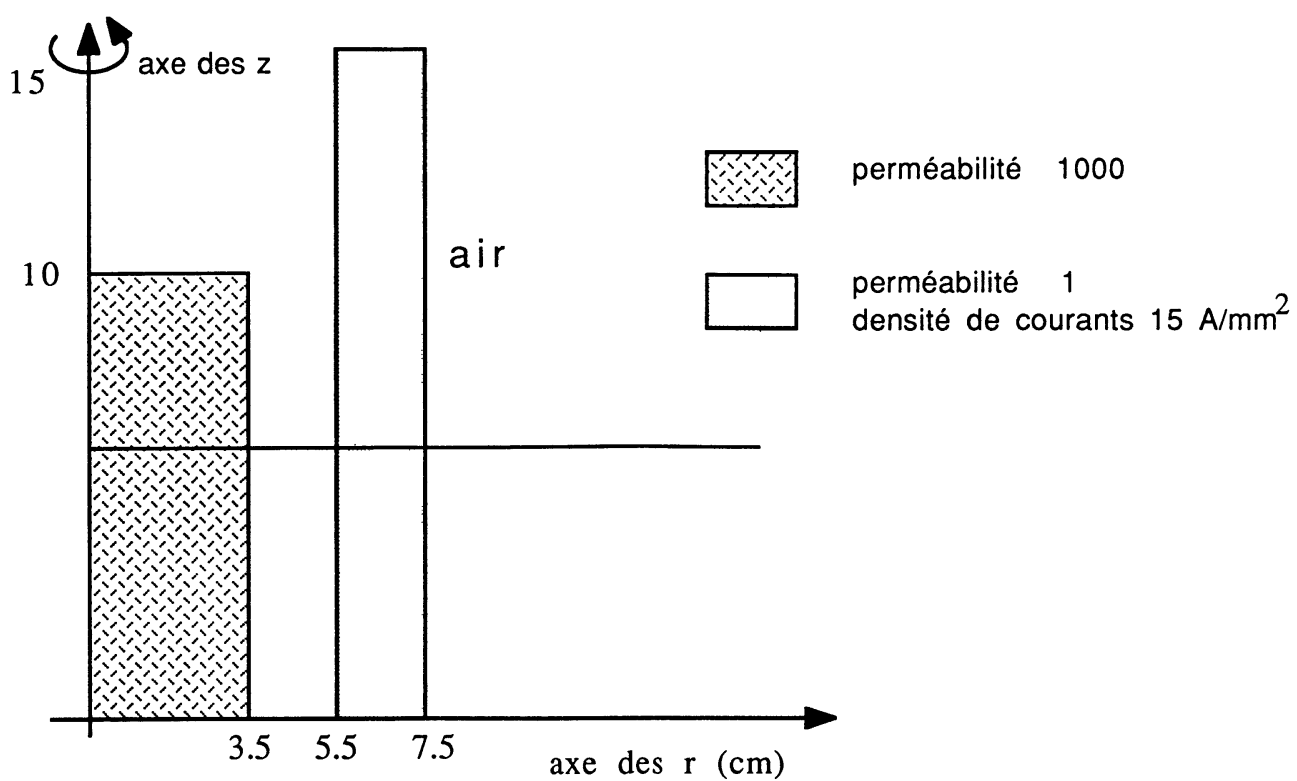

Fig. 9. - Description du problème traité.

[Illustration of the solved problem.]

ligne composée de termes tous nuls : il faut donc l'éliminer en remarquant que $(r A)$ et $\left(r H_{\mathrm{t}}\right)$ valent zéros sur l'axe.

On remarquera que l'intérêt de la formulation $(r A)$ est triple lors du couplage par rapport à la formulation $(A)$ :

- légère simplification de la formulation intégrale de frontières seule seule

- simplification de la formulation éléments finis

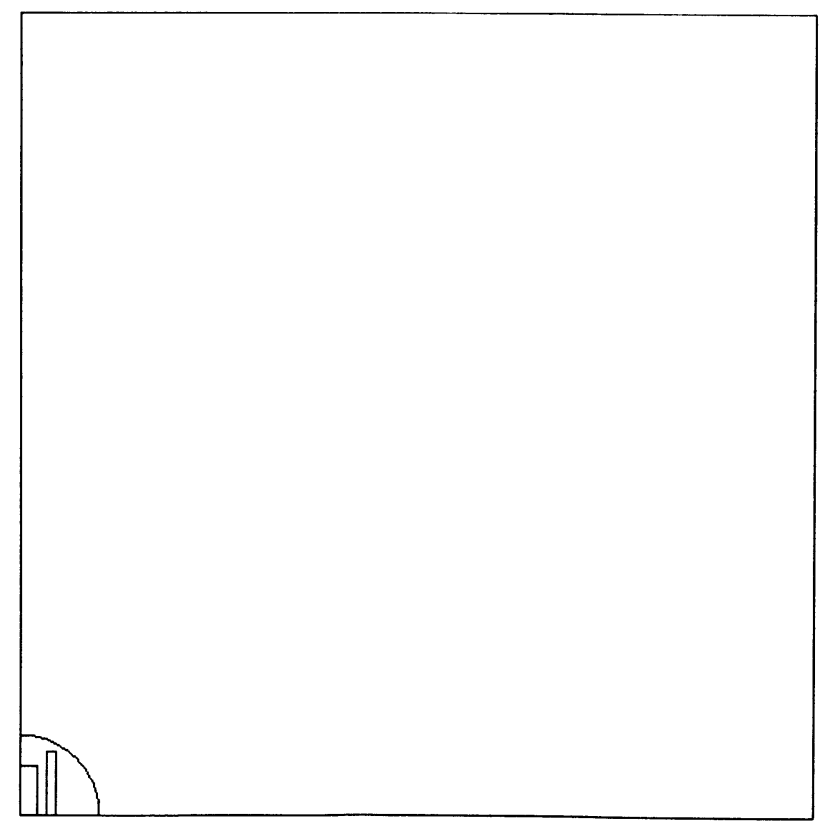

Fig. 10. - Domaine d'étude en modélisant l'infini par conditions aux limites.

[Studying area when modelling the infinite by boundary conditions.]
- simplification du couplage en lui-même, puisqu'il ne rajoute sur la frontière pour la région traitée par éléments finis, qu'un terme en $r H_{\mathrm{t}}$ (alors que la formulation en $A$ donne deux termes en $A$ et $\mathrm{d} A / \mathrm{d} n)$.

7.4 EXEMPLE ET COMPARAISON AVEC UNE SOLUTION DONNÉE PAR DES CONDITIONS AUX LIMITES. Le problème est décrit par la figure 9. Pour le modéliser par éléments finis seuls, on impose des

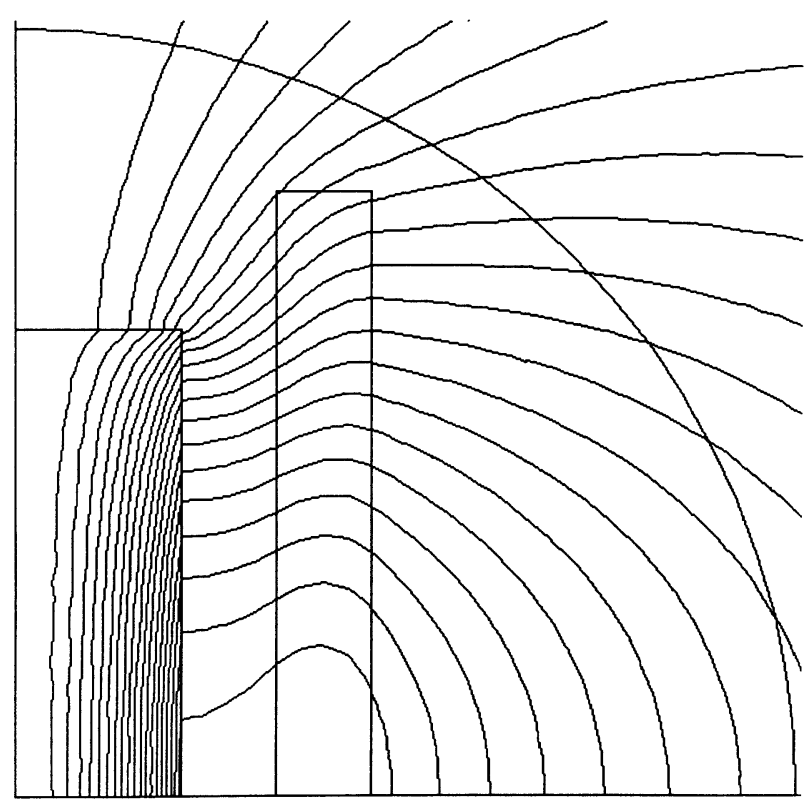

Fig. 11. - Equipotentielles $(r A)$ données par éléments finis.

[ $(r A)$ equipotential lines given by finite element analysis.] 


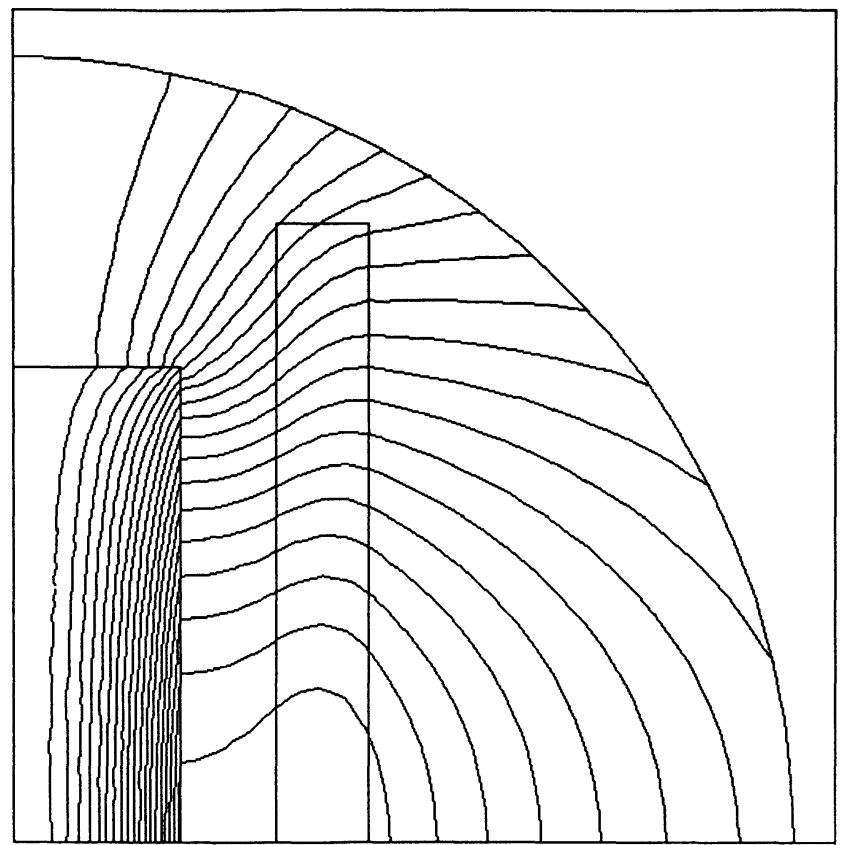

Fig. 12. - Equipotentielles $(r A)$ données par le couplage (interface dans l'air).

[ $(r A)$ equipotential lines given by the hybrid method (interface in the air).]

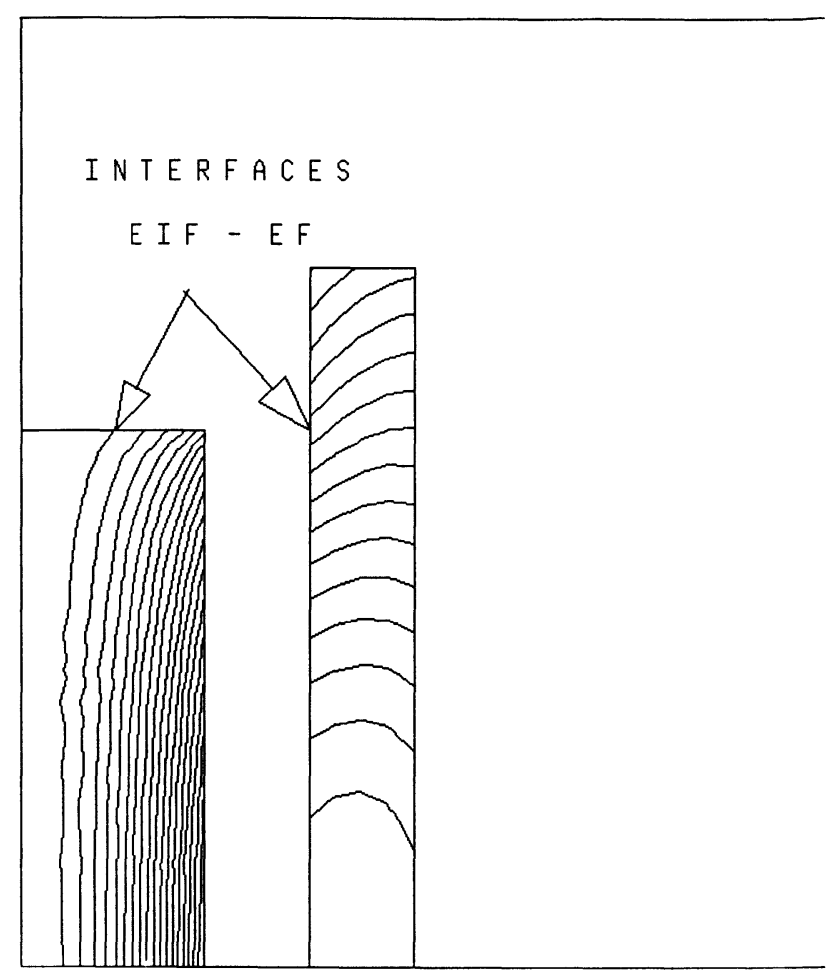

Fig. 13. - Equipotentielles $(r A)$ données par le couplage (interfaces entre les deux méthodes étant également interfaces entre régions physiques).

$[(r A)$ equipotential lines given by the hybrid method (interfaces between both methods are also interfaces between physical regions).]

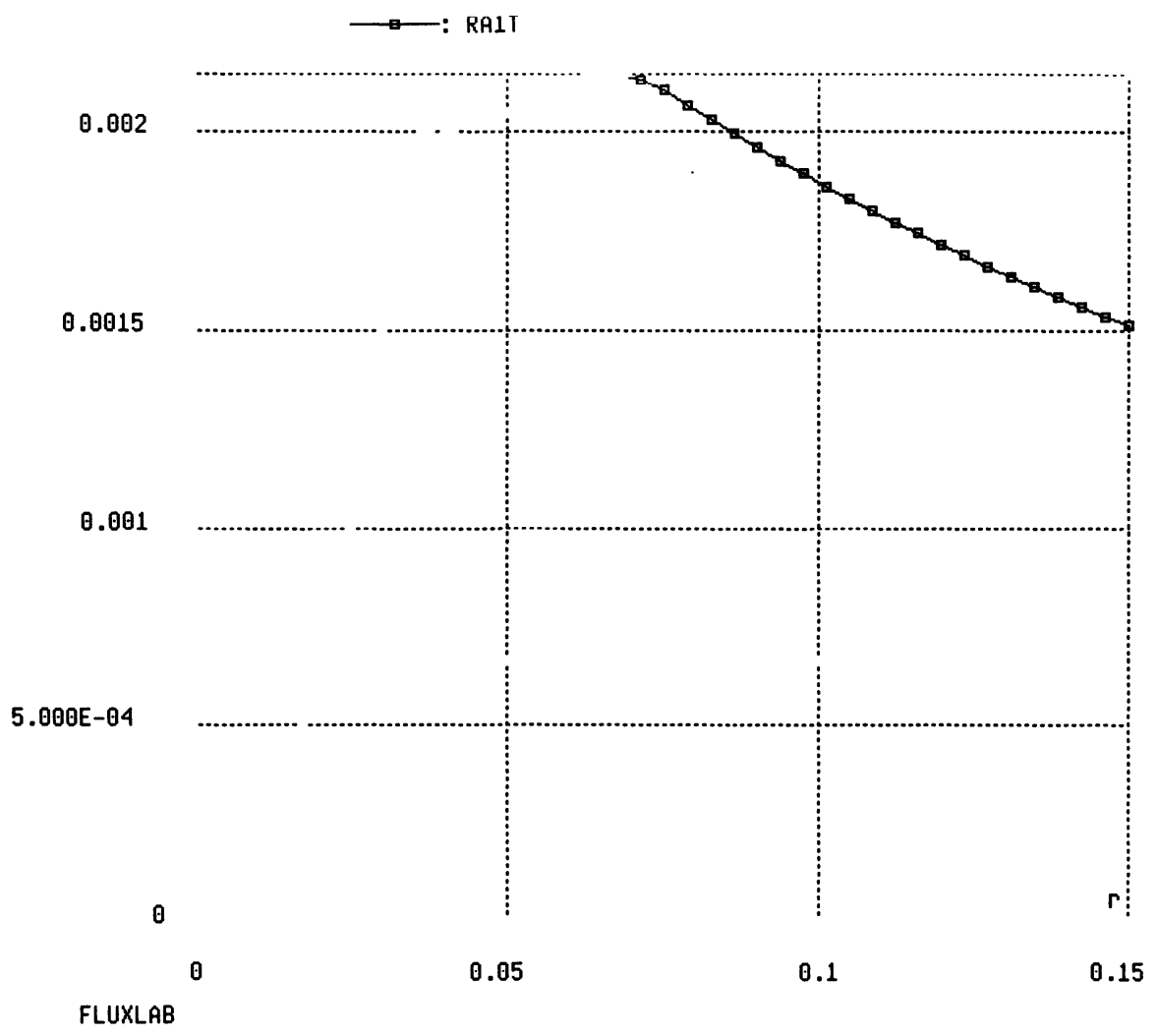

Fig. 14. - Potential $(r A)$ donné par éléments finis sur le segment.

[ $(r A)$ potential given by finite element analysis on the segment.] 


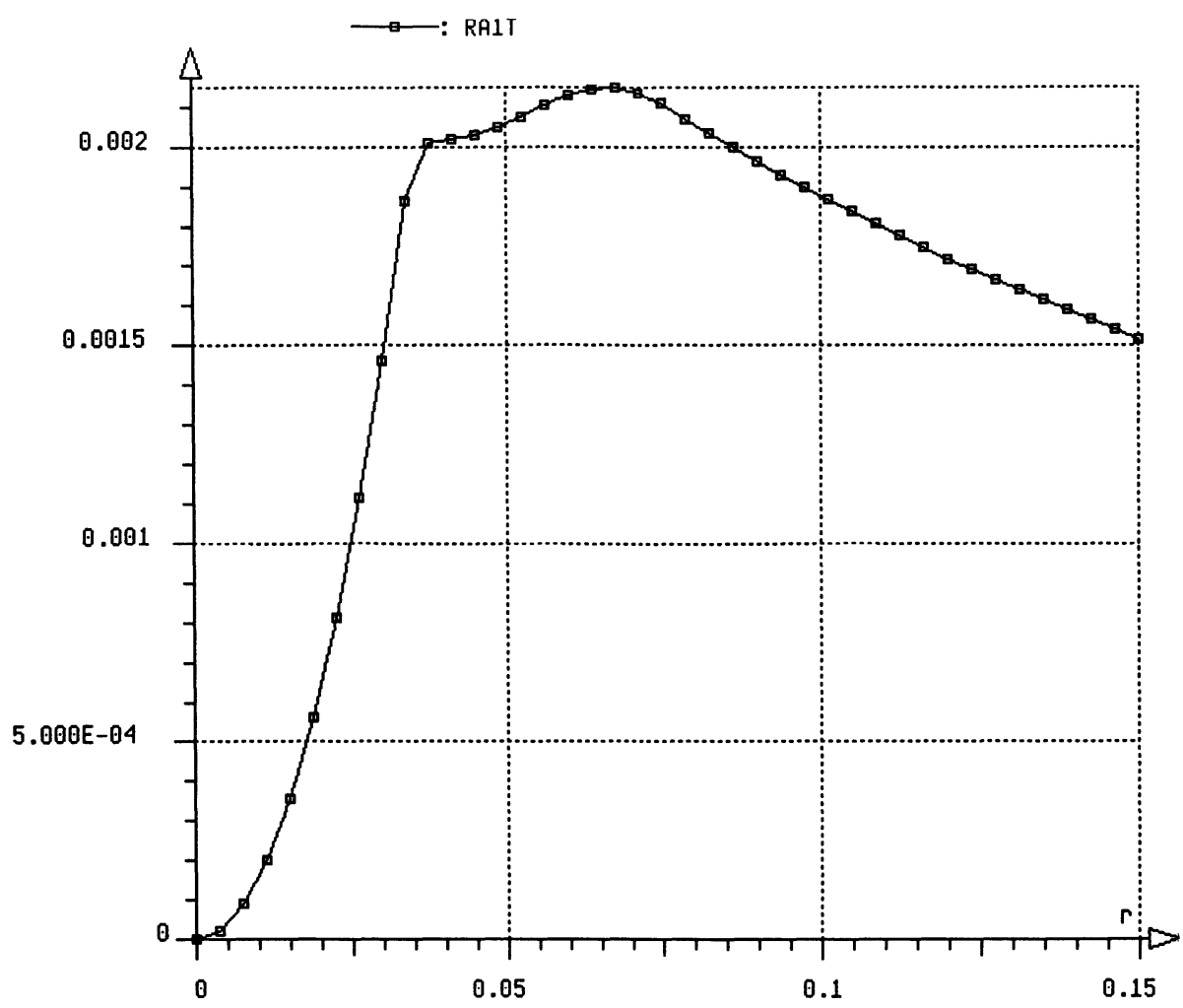

FLUXLAB

Fig. 15. - Potentiel $(r A)$ donné par la méthode couplée.

[ $(r A)$ potential given by the hybrid method.]

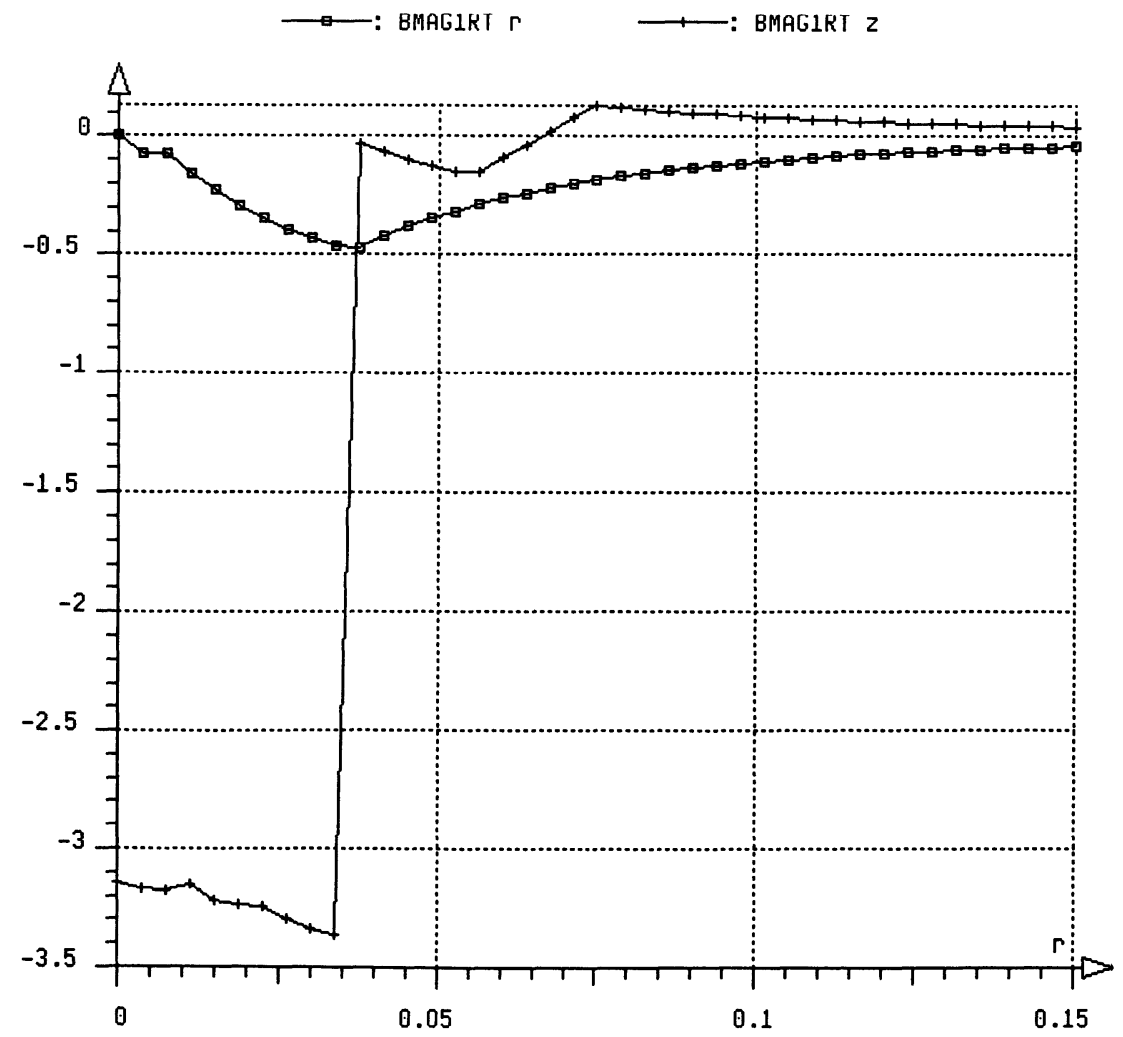

FLUXLAB

Fig. 16. - Champ donné par éléments finis sur le segment.

[Flux density given by finite element analysis on the segment.] 


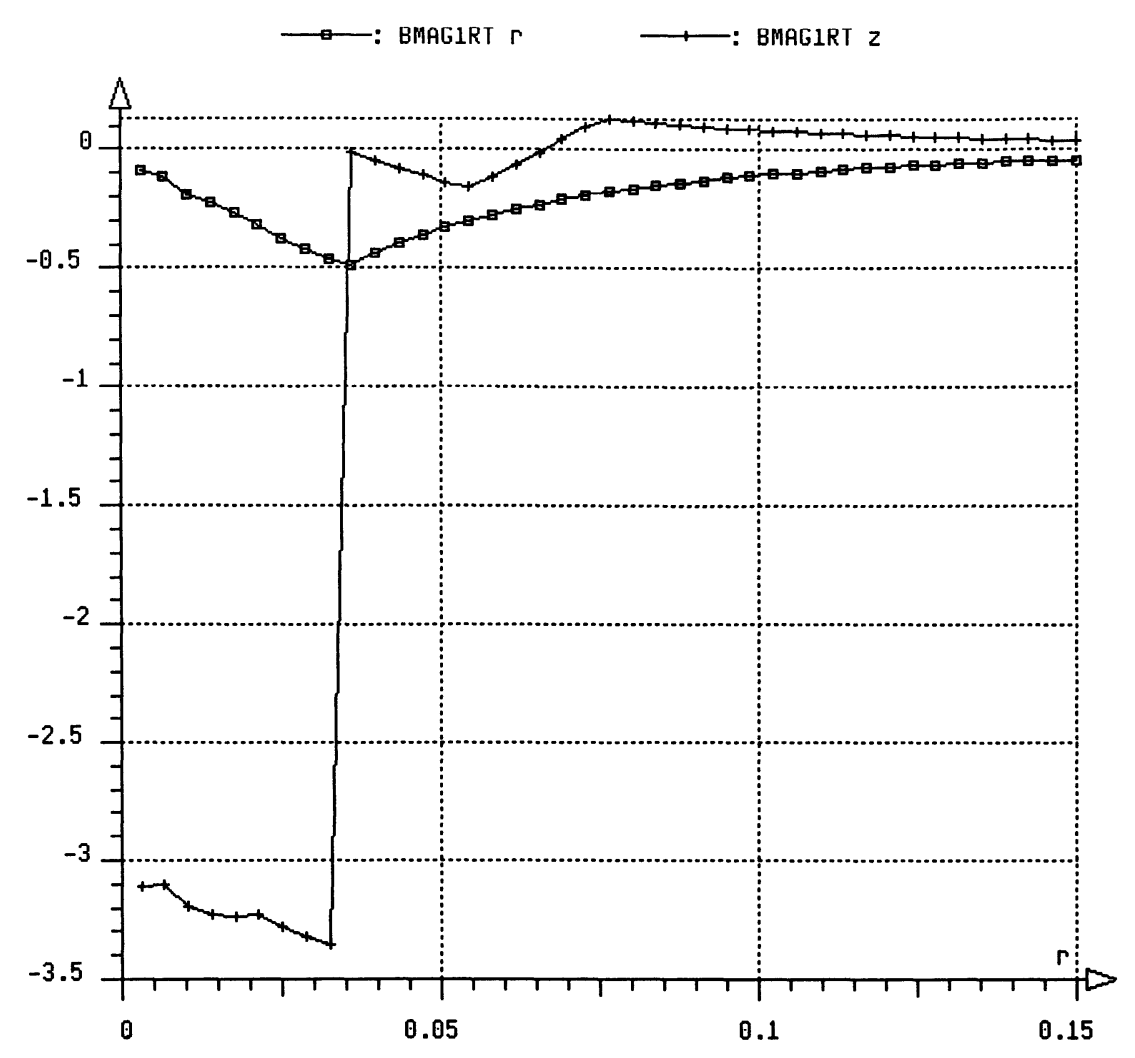

FLUXLAB

Fig. 17. - Champ donné par le couplage.

[Flux density given by the hybrid method.]

conditions aux limites de type Dirichlet $(A=0)$ et Neumann homogène sur des frontières suffisamment éloignées des sources (Fig. 10). Les figures 11, 12, 13 montrent les équipotentielles calculées par éléments finis seuls (Fig. 11), par EF-EIF avec interface entre les deux méthodes dans l'air (Fig. 12) et EF-EIF avec interfaces étant aussi interfaces entre régions physiques (Fig. 13). Les figures 14, 15 (resp. 16, 17) donnent le potentiel $r A$ (resp. le champ) sur le segment droit de la figure 9 calculé par les méthodes EF seule et EF-EIF avec interface dans l'air; la méthode EF-EIF avec interface sur les matériaux donnant des résultats moins bons (différence $=4 \%$ ) à cause de la complexité de la frontière (on a tou ours intérêt à choisir l'interface la plus simple géométriquement et physiquement).

\section{Conclusion.}

Nous avons présenté les formulations intégrales de frontières des problèmes axisymétriques pour potentiels scalaires (total ou réduit) et vecteur avec en particulier la formulation en $(r A)$, et nous avons couplé ces formulations avec les équations données par la méthode des éléments finis. Les résultats présentés montrent la validité des différents couplages. L'intérêt des méthodes hybrides est de concilier les points forts des deux méthodes: non-linéarité des matériaux pour les éléments finis, prise en compte de l'infini, non-évolution du maillage lors de déplacement de pièces mobiles pour les intégrales de frontières. Des travaux restent cependant à faire pour améliorer le système matriciel o tenu.

\section{Bibliographie}

[1] Meunier G., Coulomb J. L.., Salon S., KrähenBÜHL L., Hybrid finite element solutions for three dimensional scalar potential problems, IEEE Trans-Mag 22 (1986) 1040.
[2] Bouillault F., RazeK A., Utilisation d'une formulation mixte (éléments finis-intégrales de frontières) pour modéliser les systèmes électromagnétiques, Revue Phys. Appl. 21 (1986) 595. 
[3] Salon S. J., The hybrid finite element-boundary element method in electromagnetics, IEEE Trans-Mag 21 (1829) 1985.

[4] Bossavit A., Vérité J. C., The TRIFOU CODE : Solving the $3 \mathrm{D}$ eddy currents problem by using $\mathrm{H}$ as state variable, IEEE 83 Trans-Mag Mag 19, p. 2465 .

[5] Ren Z., Bouillault F., Razek A., Bossavit A., VÉRITÉ J. C., A new hybrid model using electric field formulation for 3D eddy currents problems, COMPUMAG 89 (Tokyo).

[6] Pichon L., RAzeK A., Force calculation in axisymmetric induction devices using a hybrid FEMBEM technique, COMPUMAG 89 (Tokyo).

[7] Pichon L., Razek A., A hybrid finite element method-boundary element method using time stepping for eddy currents calculation in axisymmetric problems, Proc. IEE P.A. 136 (1989) 217-222.

[8] KRÄHENBÜHL L., Nicolas A., Efficient techniques for boundary integral equation methods, IEEE Trans-Mag. 83.

[9] KRÄHENBÜHL L., La méthode des équations intégrales de frontière pour la résolution des problèmes de potentiel en électrotechnique, et sa formulation axisymétrique, Thèse E.C.L. 83-13, déc. 1983.

[10] Brunotte X., Meunier G., Hybrid Finite Elements/Boundary Equations Method for the analysis of magnetic fields created by complex geometries in a uniform external field, EUROBEM 90 Nice (May 1990).

[11] Durand E., Magnetostatique (Masson, Paris) 1968. 